\title{
A Four-Stage Fifth-Order Trigonometrically Fitted Semi-Implicit Hybrid Method for Solving Second-Order Delay Differential Equations
}

\author{
Sufia Zulfa Ahmad, ${ }^{1}$ Fudziah Ismail, ${ }^{1,2}$ and Norazak Senu ${ }^{1,2}$ \\ ${ }^{1}$ Department of Mathematics, Faculty of Science, Universiti Putra Malaysia, 43400 Serdang, Selangor, Malaysia \\ ${ }^{2}$ Institute for Mathematical Research, Universiti Putra Malaysia, 43400 Serdang, Selangor, Malaysia \\ Correspondence should be addressed to Fudziah Ismail; fudziah_i@yahoo.com.my
}

Received 23 February 2016; Accepted 26 April 2016

Academic Editor: Fazal M. Mahomed

Copyright ( 2016 Sufia Zulfa Ahmad et al. This is an open access article distributed under the Creative Commons Attribution License, which permits unrestricted use, distribution, and reproduction in any medium, provided the original work is properly cited.

We derived a two-step, four-stage, and fifth-order semi-implicit hybrid method which can be used for solving special second-order ordinary differential equations. The method is then trigonometrically fitted so that it is suitable for solving problems which are oscillatory in nature. The methods are then used for solving oscillatory delay differential equations. Numerical results clearly show the efficiency of the new method when compared to the existing explicit and implicit methods in the scientific literature.

\section{Introduction}

Research reveals that things depend on not only the current state of a system but also the past states, resulting in differential equations with a time delay. This kind of equations is called delay differential equations (DDEs) where the derivative at any time depends on the solution at prior times and is best known as model that incorporates past history. It is a more realistic model which includes some of the past history of the system to determine the future behavior. In this paper, we deal with numerical method for solving secondorder delay differential equations (DDEs) with constant delay which can be written in the form of

$$
\begin{array}{ll}
y^{\prime \prime}(t)=f(t, y(t), y(t-\tau)), & a \leq t \leq b, \\
y\left(t_{0}\right)=y_{0}, & \\
y^{\prime}\left(t_{0}\right)=y_{0}^{\prime}, & t \in[-\tau, a],
\end{array}
$$

where $\tau$ is the delay term and the first derivative does not appear explicitly. There are many applications which are well known related to DDEs such as population dynamics, epidemiology, and reforestation.
Equation (1) can be solved using methods such as direct multistep method, Runge-Kutta Nyström (RKN) method, and hybrid method. There has been a growing interest in the field of DDEs; Kuang [1] in his book discussed delay differential equations with applications in population dynamics. Bt Ismail and Suleiman [2] studied the P-Stability and QStability of singly diagonally implicit Runge-Kutta method for delay differential equations. Taiwo and Odetunde [3] studied delay differential equations using a decomposition method. Ismail et al. [4] used Runge-Kutta method and Hermite interpolation to solve delay differential equations. Hoo et al. [5] constructed a direct Adams-Moulton Method for solving second-order delay differential equations. Some other authors also derived block multistep method to solve delay differential equations; such work can be seen in San et al. [6], Radzi et al. [7], and Ishak et al. [8].

In our previous work Ahmad et al. [9] have derived semi-implicit hybrid method of four stages and fifth order denoted as SIHM4(5), where we incorporated the phase-lag and amplification equations, in the derivation, so that we obtained method which has higher order of dissipation and dispersion. But in this paper, we are going to construct a new semi-implicit hybrid method (NSIHM) of four stages and fifth order using the algebraic order conditions given 
in Coleman [10]; the derivation also incorporates the simplifying conditions as well as the technique of minimization of the error constant. The method is then trigonometrically fitted using similar approach as in [11, 12] so that it has a higher order of dissipation and dispersion; this approach is simpler than incorporating the phase-lag and amplification equations into the derivation. In all the previous work regarding phase-fitted methods, the methods are used to solve oscillatory second-order ordinary differential equations (ODEs). Here the trigonometrically fitted method is used for solving retarded second-order delay differential equations which are oscillatory in nature. The efficiency of the new method will be compared with several other existing explicit and implicit methods of RKN type and hybrid methods.

\section{Derivation of Four-Stage Fifth-Order Semi-Implicit Hybrid Methods}

An $s$-stage semi-implicit hybrid method for the numerical integration of the IVPs is given as

$$
\begin{aligned}
Y_{i} & =\left(1+c_{i}\right) y_{n}-c_{i} y_{n-1}+h^{2} \sum_{j=1}^{s} a_{i j} f\left(t_{n}+c_{j} h, Y_{j}\right), \\
y_{n+1} & =2 y_{n}-y_{n-1}+h^{2} \sum_{i=1}^{s} b_{i} f\left(t_{n}+c_{i} h, Y_{i}\right),
\end{aligned}
$$

where $i=1, \ldots, s$ and $i \geq j$. The equations of form (2) are defined as

$$
\begin{aligned}
Y_{1}= & y_{n-1}, \\
Y_{2}= & y_{n}, \\
Y_{i}= & \left(1+c_{i}\right) y_{n}-c_{i} y_{n-1}+h^{2} \sum_{j=1}^{i} a_{i j} f\left(t_{n}+c_{j} h, Y_{j}\right), \\
y_{n+1}= & 2 y_{n}-y_{n-1} \\
& +h^{2}\left(b_{1} f_{n-1}+b_{2} f_{n}+\sum_{i=3}^{s} b_{i} f\left(t_{n}+c_{i} h, Y_{i}\right)\right),
\end{aligned}
$$

where the first two nodes are $c_{1}=-1$ and $c_{2}=0$ and $i=$ $3, \ldots, s$, while functions $f_{n-1}=f\left(t_{n-1}, y_{n-1}\right)$ and $f_{n}=f\left(t_{n}\right.$, $\left.y_{n}\right)$. The coefficients of $b_{i}, c_{i}$, and $a_{i j}$ can be represented in Butcher tableau as follows:

$$
\begin{array}{c|ccccc}
-1 & 0 & & & & \\
0 & 0 & 0 & & & \\
c_{3} & a_{3,1} & a_{3,2} & \gamma & & \\
\vdots & \vdots & \vdots & \ddots & \ddots & \\
c_{s} & a_{s, 1} & a_{s, 2} & \cdots & a_{s, s-1} & \gamma \\
\hline & b_{1} & b_{2} & \cdots & b_{s-1} & b_{s}
\end{array}
$$

The coefficients of the diagonal element $(\gamma)$ are always equal for this method. Here, we derive the four-stage fifthorder NSIHM based on the order conditions, simplifying conditions, and minimization of the error constant $C_{p+1}$ of the method. The error constant is defined by

$$
C_{p+1}=\left\|\left(e_{p+1}\left(t_{1}\right)\right), \ldots, e_{p+1}\left(t_{k}\right)\right\|_{2},
$$

where $k$ is the number of trees of order $p+2\left(p\left(t_{i}\right)=p+2\right)$ and $e_{p+1}\left(t_{i}\right)$ is the local truncation error defined in Coleman [10]. The order conditions defined in Coleman [10] for hybrid method up to order six are listed as follows:

(i) Order 2:

$$
\sum_{i=1}^{s} b_{i}=1
$$

(ii) Order 3:

$$
\sum_{i=1}^{s} b_{i} c_{i}=0
$$

(iii) Order 4:

$$
\begin{aligned}
& \sum_{i=1}^{s} b_{i} c_{i}^{2}=\frac{1}{6}, \\
& \sum_{i=1}^{s} b_{i} a_{i j}=\frac{1}{12} .
\end{aligned}
$$

(iv) Order 5:

$$
\begin{gathered}
\sum_{i=1}^{s} b_{i} c_{i}^{3}=0, \\
\sum_{i=1}^{s} b_{i} c_{i} a_{i j}=\frac{1}{12}, \\
\sum_{i=1}^{s} b_{i} a_{i j} c_{j}=0 .
\end{gathered}
$$

(v) Order 6:

$$
\begin{aligned}
\sum_{i=1}^{s} b_{i} c_{i}{ }^{4} & =\frac{1}{15}, \\
\sum_{i=1}^{s} b_{i} c_{i}^{2} a_{i j} & =\frac{1}{30}, \\
\sum_{i=1}^{s} b_{i} c_{i} a_{i j} c_{j} & =-\frac{1}{60}, \\
\sum_{i=1}^{s} b_{i} a_{i j} a_{i k} & =\frac{7}{120}, \\
\sum_{i=1}^{s} b_{i} a_{i j} c_{j}^{2} & =\frac{1}{180}, \\
\sum_{i=1}^{s} b_{i} a_{i j} a_{j k} & =\frac{1}{360},
\end{aligned}
$$


where value of $i \geq j \geq k$. For $c_{i}$, the method needs to satisfy the simplifying condition which is

$$
\sum_{i}^{s} a_{i j}=\frac{\left(c_{i}^{2}+c_{i}\right)}{2}, \text { for } i=3, \ldots, s .
$$

First, we derive the four-stage fifth-order NSIHM using the algebraic order conditions up to order five (see (6) to (9)) and simplifying condition in (11). We obtained the solution for the coefficients in terms of $a_{41}, a_{43}, a_{44}, b_{4}$, and $c_{4}$ listed as follows:

$$
\begin{aligned}
& a_{31}=\frac{K}{\left(-1+6 b_{4} c_{4}+6 b_{4} c_{4}{ }^{2}\right)^{4}}, \\
& a_{32}=\frac{M}{\left(-1+6 b_{4} c_{4}+6 b_{4} c_{4}^{2}\right)^{4}}, \\
& a_{42}=-a_{41}-a_{43}-a_{44}+\frac{c_{4}{ }^{2}}{2}+\frac{c_{4}}{2}, \\
& b_{1}=\frac{-1+12 b_{4} c_{4}^{2}}{12\left(-1+3 b_{4} c_{4}+6 b_{4} c_{4}{ }^{2}+3 b_{4} c_{4}^{3}\right)}, \\
& b_{2}=\frac{-5+6 b_{4}-6 b_{4} c_{4}+24 b_{4} c_{4}{ }^{2}+36 b_{4} c_{4}^{3}}{6\left(-1+6 b_{4} c_{4}{ }^{3}+6 b_{4} c_{4}{ }^{2}\right)}, \\
& b_{3} \\
& =\frac{\left(-1+6 b_{4} c_{4}+6 b_{4} c_{4}^{2}\right)^{3}}{12\left(-1+3 b_{4} c_{4}+6 b_{4} c_{4}{ }^{2}+3 b_{4} c_{4}{ }^{3}\right)\left(-1+6 b_{4} c_{4}{ }^{3}+6 b_{4} c_{4}^{2}\right)}, \\
& c_{3}=\frac{-1+6 b_{4} c_{4}{ }^{3}+6 b_{4} c_{4}^{2}}{-1+6 b_{4} c_{4}+6 b_{4} c_{4}{ }^{2}},
\end{aligned}
$$

where

$$
\begin{aligned}
K & =\left(-1+6 b_{4} c_{4}{ }^{3}+6 b_{4} c_{4}{ }^{2}\right)\left(6 b_{4} a_{44} c_{4}+12 b_{4} a_{41}\right. \\
& -12 b_{4} a_{43}-a 44+18 b_{4} a_{44} c_{4}{ }^{2}-72 b_{4}{ }^{2} a_{44} c_{4}{ }^{3} \\
& -72 b_{4}{ }^{2} a_{44} c_{4}{ }^{4}-144 b_{4}{ }^{2} a_{41} c_{4}{ }^{2}+216 b_{4}{ }^{3} a_{41} c_{4}{ }^{2} \\
& +648 b_{4}{ }^{3} a_{41} c_{4}{ }^{3}+648 b_{4}{ }^{3} a_{41} c_{4}{ }^{4}-36 b_{4}{ }^{2} a_{41} c_{4}{ }^{3} \\
& +216 b_{4}{ }^{3} a_{41} c_{4}{ }^{5}+144 b_{4}{ }^{2} a_{43} c_{4}{ }^{2}+108 b_{4}{ }^{2} a_{43} c_{4}{ }^{3} \\
& -648 b_{4}{ }^{3} a_{43} c_{4}{ }^{4}-648 b_{4}{ }^{3} a_{43} c_{4}{ }^{5}-216 b_{4}{ }^{3} a_{43} c_{4}{ }^{6} \\
& \left.-216 b_{4}{ }^{3} a_{43} c_{4}{ }^{3}-108 b_{4}{ }^{2} a_{41} c_{4}+36 b_{4}{ }^{2} a_{43} c_{4}\right), \\
M & =-\left(-1-30 b_{4} a_{44} c_{4}+15 b_{4} c_{4}+24 b_{4} c_{4}{ }^{2}-12 b_{4} a_{41}\right. \\
& +12 b_{4} a_{43}+9 b_{4} c_{4}{ }^{2}+2 a_{44}-6 b_{4} a_{44} c_{4}{ }^{3} \\
& +108 b_{4}{ }^{2} a_{44} c_{4}{ }^{5}-432 b_{4}{ }^{3} a_{44} c_{4}{ }^{7}+5184 b_{4}{ }^{4} a_{41} c_{4}{ }^{5} \\
& +7776 b_{4}{ }^{4} a_{41} c_{4}{ }^{6}+5184 b_{4}{ }^{4} a_{41} c_{4}{ }^{7}-216 b_{4}{ }^{3} a_{41} c_{4}{ }^{6} \\
& +1296 b_{4}{ }^{4} a_{41} c_{4}{ }^{8}-7776 b_{4}{ }^{4} a_{43} c_{4}{ }^{7}-5184 b_{4}{ }^{4} a_{43} c_{4}{ }^{8} \\
& -1296 b_{4}{ }^{4} a_{43} c_{4}{ }^{9}-5184 b_{4}{ }^{4} a_{43} c_{4}{ }^{6}+1296 b_{4}{ }^{4} a_{41} c_{4}{ }^{4}
\end{aligned}
$$

$$
\begin{aligned}
& -1296 b_{4}{ }^{4} a_{43} c_{4}{ }^{5}+1296 b_{4}{ }^{4} a_{44} c_{4}{ }^{4}+5184 b_{4}{ }^{4} a_{44} c_{4}{ }^{5} \\
& +7776 b_{4}{ }^{4} a_{44} c_{4}{ }^{6}+5184 b_{4}{ }^{4} a_{44} c_{4}{ }^{7}+1296 b_{4}{ }^{4} a_{44} c_{4}{ }^{8} \\
& -48 b_{4} a_{44} c_{4}{ }^{2}+216 b_{4}{ }^{2} a_{44} c_{4}{ }^{2}+540 b_{4}{ }^{2} a_{44} c_{4}{ }^{3} \\
& +432 b_{4}{ }^{2} a_{44} c_{4}{ }^{4}-864 b_{4}{ }^{3} a_{44} c_{4}{ }^{3}-2592 b_{4}{ }^{3} a_{44} c_{4}{ }^{4} \\
& -3024 b_{4}{ }^{3} a_{44} c_{4}{ }^{5}-1728 b_{4}{ }^{3} a_{44} c_{4}{ }^{6}+216 b_{4}{ }^{2} a_{41} c_{4}{ }^{2} \\
& -216 b_{4}{ }^{3} a_{41} c_{4}{ }^{2}-1296 b_{4}{ }^{3} a_{41} c_{4}{ }^{3}-2160 b_{4}{ }^{3} a_{41} c_{4}{ }^{4} \\
& +108 b_{4}{ }^{2} a_{41} c_{4}{ }^{3}-1296 b_{4}{ }^{3} a_{41} c_{4}{ }^{5}-216 b_{4}{ }^{2} a_{43} c_{4}{ }^{2} \\
& -180 b_{4}{ }^{2} a_{43} c_{4}{ }^{3}+1728 b_{4}{ }^{3} a_{43} c_{4}{ }^{4}+2160 b_{4}{ }^{3} a_{43} c_{4}{ }^{5} \\
& +864 b_{4}{ }^{3} a_{43} c_{4}{ }^{6}+432 b_{4}{ }^{3} a_{43} c_{4}{ }^{3}-72 b_{4}{ }^{2} c_{4}{ }^{2} \\
& -270 b_{4}{ }^{2} c_{4}{ }^{3}-342 b_{4}{ }^{2} c_{4}{ }^{4}+108 b_{4}{ }^{3} c_{4}{ }^{3}+864 b_{4}{ }^{3} c_{4}{ }^{4} \\
& +2160 b_{4}{ }^{3} c_{4}{ }^{5}+2376 b_{4}{ }^{3} c_{4}{ }^{6}-162 b_{4}{ }^{2} c_{4}{ }^{5}-18 b_{4}{ }^{2} c_{4}{ }^{6} \\
& -648 b_{4}{ }^{4} c_{4}{ }^{5}-3240 b_{4}{ }^{4} c_{4}{ }^{6}-6480 b_{4}{ }^{4} c_{4}{ }^{7} \\
& -6480 b_{4}{ }^{4} c_{4}{ }^{8}+1188 b_{4}{ }^{3} c_{4}{ }^{7}+216 b_{4}{ }^{3} c_{4}{ }^{8} \\
& -3240 b_{4}{ }^{4} c_{4}{ }^{9}-648 b_{4}{ }^{4} c_{4}{ }^{10}+108 b_{4}{ }^{2} a_{41} c_{4} \\
& \left.{ }^{2} a_{43} c_{4}\right) \\
& +
\end{aligned}
$$

By minimizing the error norm and letting the values of $a_{41}=$ $150617 / 771120, a_{43}=141097 / 771120, a_{44}=1 / 81, b_{4}=1 /$ 81 , and $c_{4}=1$, we obtained the other coefficients of the fourstage fifth-order semi-implicit hybrid method denoted as NSIHM4(5) which can be written in Butcher tableau as follows:

$$
\begin{array}{c|cccc}
-1 & 0 & & & \\
0 & 0 & 0 & & \\
1 & \frac{1}{81} & \frac{79}{81} & \frac{1}{81} & \\
1 & \frac{150617}{771120} & \frac{234943}{385560} & \frac{141097}{771120} & \frac{1}{81} \\
\hline & \frac{1}{12} & \frac{5}{6} & \frac{23}{324} & \frac{1}{81}
\end{array}
$$

The norm of the principal local truncation error coefficient for $y_{n}$ is given by

$$
\left\|\tau^{(6)}\right\|_{2}=1.1472 \times 10^{-1},
$$

where $\left\|\tau^{(6)}\right\|_{2}$ is the norm of the error equations for the sixthorder method.

\section{Trigonometrically Fitting the Semi-Implicit Hybrid Method}

To trigonometrically fit the new method NSIHM4(5), we consider stage three and stage four of the NSIHM4(5) in (14). 
The new method which will be derived is denoted as fourstage fifth-order trigonometrically fitted semi-implicit hybrid method or TF-NSIHM4(5) which is of fifth algebraic order which is the same as the algebraic order of NSIHM4(5). Note that trigonometrically fitting the method will not change the algebraic order of the method. The method can be written in Butcher tableau as follows:

$$
\begin{array}{c|cccc}
-1 & 0 & & & \\
0 & 0 & 0 & & \\
1 & \frac{1}{81} & a_{32} & \frac{1}{81} & \\
1 & \frac{150617}{771120} & a_{42} & \frac{141097}{771120} & \frac{1}{81} \\
\hline & b_{1} & b_{2} & b_{3} & \frac{1}{81}
\end{array}
$$

The values of $a_{32}, a_{42}, b_{1}, b_{2}$, and $b_{3}$ are modified using the trigonometrically fitting technique so that it would improve the accuracy of the method and suitable for solving oscillatory problems.

We require the internal stage (stages 3 and 4) and the updating stage to integrate exactly the linear combination of the functions $\{\sin (v t), \cos (v t)\}$ for $v \in \mathscr{R}$ subject to the fifthorder formulae. Hence, we obtain the following equations:

$$
\begin{aligned}
& \cos \left(c_{3} H\right)=1+c_{3}-c_{3} \cos (H)-H^{2}\left\{a_{31} \cos (H)\right. \\
& \left.\quad+a_{32}+a_{33} \cos \left(c_{3} H\right)\right\}, \\
& \sin \left(c_{3} H\right)=c_{3} \sin (H)+H^{2}\left\{a_{31} \sin (H)\right. \\
& \left.\quad-a_{33} \sin \left(c_{3} H\right)\right\}, \\
& \cos \left(c_{4} H\right)=1+c_{4}-c_{4} \cos (H)-H^{2}\left\{a_{41} \cos (H)\right. \\
& \left.\quad+a_{42}+a_{43} \cos \left(c_{3} H\right)+a_{44} \cos \left(c_{4} H\right)\right\}, \\
& \sin \left(c_{4} H\right)=c_{4} \sin (H)+H^{2}\left\{a_{41} \sin (H)\right. \\
& \left.\quad-a_{43} \sin \left(c_{3} H\right)-a_{44} \sin \left(c_{4} H\right)\right\}, \\
& 2 \cos (H)=2-H^{2}\left\{b_{1} \cos (H)+b_{2}+b_{3} \cos \left(c_{3} H\right)\right. \\
& \left.\quad+b_{4} \cos \left(c_{4} H\right)\right\}, \\
& b_{1} \sin (H)=b_{3} \sin \left(c_{3} H\right)+b_{4} \sin \left(c_{4} H\right) .
\end{aligned}
$$

Solving (17) to (20) with the choice of coefficients $c_{3}=1, c_{4}=$ $1, a_{33}=1 / 81, a_{41}=150617 / 771120, a_{43}=141097 / 771120$, and $a_{44}=1 / 81$ simultaneously, we obtained

$$
\begin{aligned}
a_{32} & =-\frac{2}{81} \frac{81 \cos (H)-81+H^{2} \cos (H)}{H^{2}}, \\
a_{42} & =-\frac{1}{385560} \\
& \frac{771120 \cos (H)-771120+150617 H^{2} \cos (H)}{H^{2}},
\end{aligned}
$$

where $=v h, h$ is step size, and $v$ is the fitted frequency. Next, using (21) and (22) and another two additional order conditions in (6) and (7) for the fifth-order method which are

$$
\begin{aligned}
& b_{1}+b_{2}+b_{3}+b_{4}=1, \\
& -b_{1}+b_{3} c_{3}+b_{4} c_{4}=0,
\end{aligned}
$$

with the choice of coefficients $c_{3}=1, c_{4}=1$, and $b_{4}=1 / 81$, we solve the equations simultaneously to get $b_{1}, b_{2}$, and $b_{3}$, which are given as follows:

$$
\begin{aligned}
& b_{1}=-\frac{1}{2} \frac{\cos (H)-2+H^{2}}{Q}, \\
& b_{2}=\frac{2 \cos (H)-2+H^{2} \cos (H)}{Q} \\
& b_{3}=-\frac{1}{162} \frac{162 \cos (H)-162+2 H^{2} \cos (H)+79 H^{2}}{Q},
\end{aligned}
$$

where

$$
Q=H^{2}(\cos (H)-1) .
$$

The above formulae can be expressed in Taylor series expansions:

$$
\begin{aligned}
a_{32}= & \frac{79}{81}-\frac{23}{324} H^{2}+\frac{17}{9720} H^{4}-\frac{5}{326592} H^{6} \\
& -\frac{1}{16329600} H^{8}+O\left(H^{10}\right) \\
a_{42}= & \frac{234943}{385560}+\frac{86357}{771120} H^{2}-\frac{124913}{9253440} H^{4} \\
& +\frac{136847}{277603200} H^{6}-\frac{142049}{15545779200} H^{8} \\
& +O\left(H^{10}\right), \\
b_{1}= & \frac{1}{12}+\frac{1}{240} H^{2}+\frac{1}{6048} H^{4}+\frac{1}{172800} H^{6} \\
& +O\left(H^{8}\right), \\
b_{2}= & \frac{5}{6}-\frac{1}{120} H^{2}-\frac{1}{3024} H^{4}-\frac{1}{86400} H^{6} \\
& +O\left(H^{10}\right), \\
b_{3}= & \frac{23}{324}+\frac{1}{240} H^{2}+\frac{1}{6048} H^{4}+\frac{1}{172800} H^{6} \\
& +O\left(H^{8}\right) \cdot
\end{aligned}
$$

The values of $a_{32}, a_{42}, b_{1}, b_{2}$, and $b_{3}$ are constants for constants $v$ and $h$ and the other coefficients remain the same.

\section{Problems Tested and Numerical Results}

In this section, the new method NSIHM4(5) and the trigonometrically fitted method, TF-NSIHM4(5), are used to solve a 
set of oscillatory delay differential equations problems. The delay terms are evaluated using Newton divided different interpolation. Numerical results are tabulated and compared with the existing explicit and implicit methods in the scientific literature. The test problems are listed as follows.

Problem 1. It is as follows:

$$
y^{\prime \prime}(t)=-\frac{1}{2} y(t)+\frac{1}{2} y(t-\pi), \quad 0 \leq t \leq 8 \pi, y_{o}=0 .
$$

The fitted frequency is $v=1$. Exact solution is $y(t)=\sin (t)$, source: Schmitt [13].

Problem 2. It is as follows:

$$
y^{\prime \prime}(t)-y(t)+\eta(t) y\left(\frac{t}{2}\right)=0, \quad 0 \leq t \leq 2 \pi
$$

where

$$
\eta(t)=\frac{4 \sin (t)}{(2-2 \cos (t))^{1 / 2}}, \quad \eta(0)=4 .
$$

Fitted frequency is $v=2$. Exact solution is $y(t)=\sin (t)$, source: Schmitt [13].

Problem 3. It is as follows:

$$
y^{\prime \prime}(t)=y(t-\pi), \quad 0 \leq t \leq 8 \pi, y_{o}=0 .
$$

Fitted frequency is $v=1$. Exact solution is $y(t)=\sin (t)$, source: Ladas and Stavroulakis [14].

Problem 4. It is as follows:

$$
y^{\prime \prime}(t)=-\frac{\sin (t)}{2-\sin (t)} y(t-\pi), \quad 0 \leq t \leq 8 \pi, y_{o}=2 .
$$

Fitted frequency is $v=1$. Exact solution is $y(t)=2+\sin (t)$, source: Singh [15].

The following notations are used in Figures 1-4:

(i) SIHM4(5): SIHM derived in Ahmad et al. [9].

(ii) NSIHM4(5): a four-stage fifth-order new semi-implicit hybrid method derived in this paper.

(iii) TF-NSIHM4(5): a four-stage fifth-order new trigonometrically fitted semi-implicit hybrid method derived in this paper.

(iv) DIRKN4(4): a four-stage fourth-order dispersive order six of DIRKN method by Senu et al. [16].

(v) MPAFRKN4(4): modified phase-fitted and amplification-fitted RKN method of four stages and fourth order by Papadopoulos et al. [17].

(vi) EHM4(5): explicit hybrid method of order five derived in Franco [18].

(vii) DIRKN3(4): a three-stage fourth-order dispersive order six of DIRKN method by Senu et al. [19].

(viii) PFRKN4(4): a phase-fitted RKN method of four stages and fourth order by Papadopoulos et al. [20].

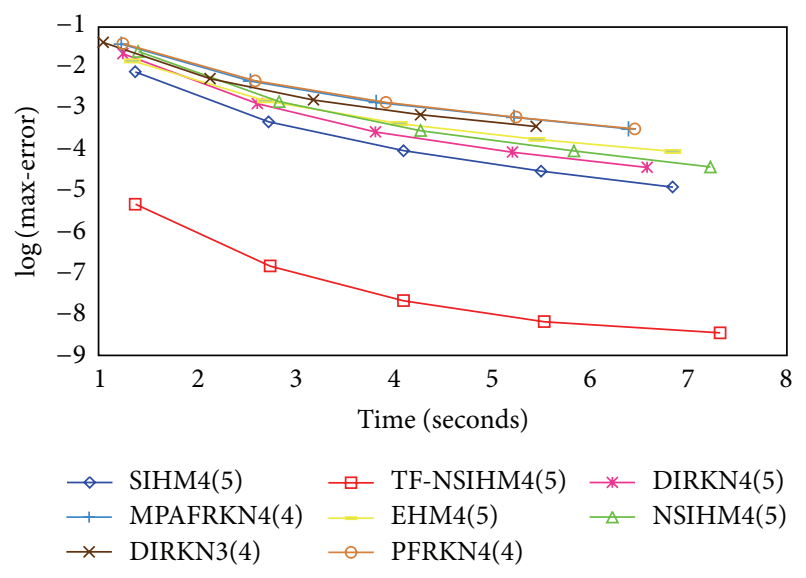

Figure 1: The efficiency curves for Problem 1 with $h=\pi / 4 i$, for $i=1, \ldots, 4$.

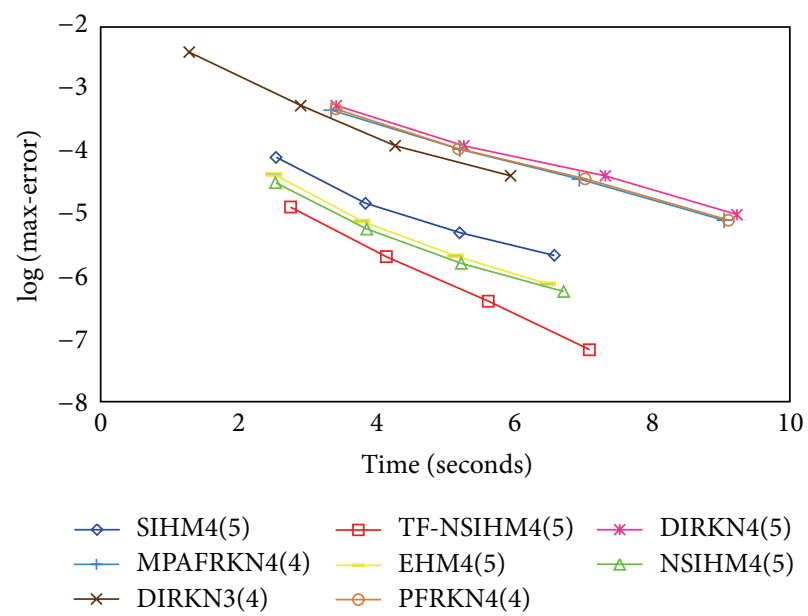

Figure 2: The efficiency curves for Problem 2 with $h=\pi / 16 i$, for $i=1, \ldots, 4$.

A measure of the accuracy is examined using absolute error which is defined by

$$
\text { Absolute error }=\max \left\{\left|y\left(t_{n}\right)-y_{n}\right|\right\},
$$

where $y\left(t_{n}\right)$ is the exact solution and $y_{n}$ is the computed solution. The efficiency curves are presented whereby the logarithms of the maximum global errors are plotted against the CPU time in seconds.

In analyzing the numerical results, methods of the same order or stage are compared. The results are given in Figures 1-4. We observed that all the methods give better accuracy for smaller step size, $h$. However the new TF-NSIHM4(5) method is the most efficient method in solving oscillatory DDEs compared to other explicit and implicit RKN and hybrid methods.

\section{Discussion and Conclusion}

In this paper we derived a four-stage fifth-order semiimplicit hybrid method. The method has a minimized local 


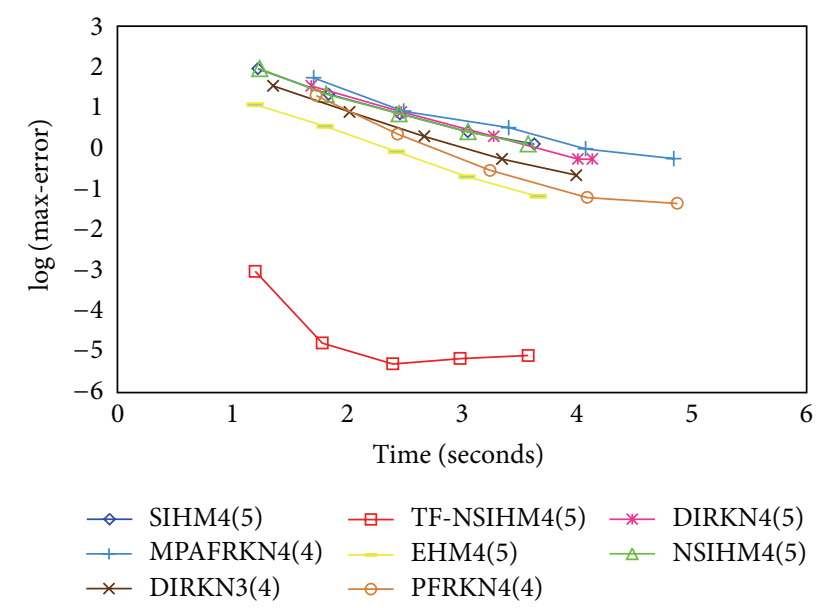

FIgUre 3: The efficiency curves for Problem 3 with $h=\pi / 2^{i}$, for $i=2, \ldots, 6$.

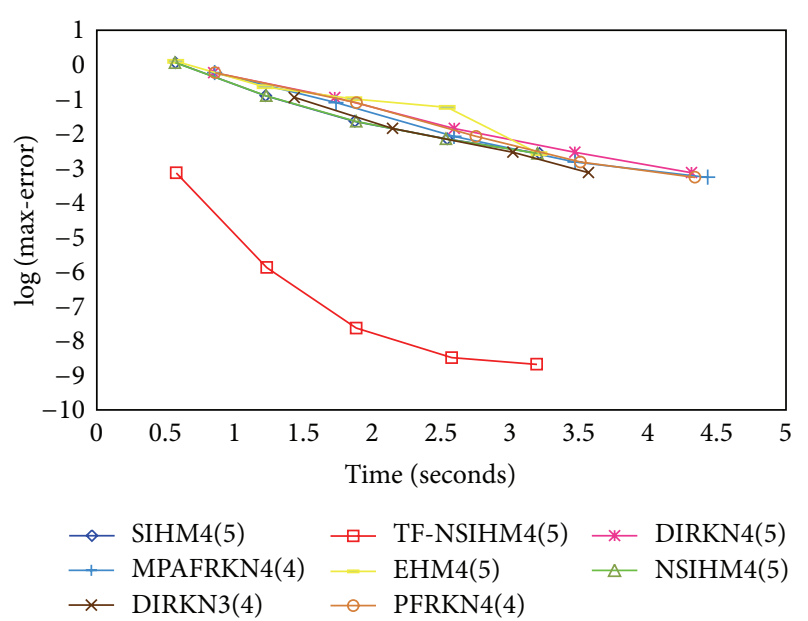

Figure 4: The efficiency curves for Problem 4 with $h=\pi / 2^{i}$, for $i=1, \ldots, 5$.

truncation error and it is suitable for directly solving special second-order ODEs. The new method is called NSIHM4(5); it is then trigonometrically fitted so that it is suitable for solving oscillatory problems and denoted as TF-NSIHM4(5).

Both methods are then used for solving oscillatory second-order DDEs; numerical results clearly have shown that the TF-NSIHM4(5) is the most efficient compared to the original NSIHM4(5) method and other existing methods in the scientific literature. Take note also that the existing methods were derived with high order of dispersion and dissipation and purposely derived for solving highly oscillatory problems. Trigonometrically fitting the method improved the efficiency of the NSIHM4(5) hybrid method and it is much easier to derive compared to the approach whereby we have to include the dispersion and dissipation equations in the derivation of the method.

Even though the new TF-NSIHM4(5) is a semi-implicit method and fairly expensive in computation time, it is still more efficient compared to the existing explicit and implicit methods.

\section{Competing Interests}

The authors declare that they have no competing interests.

\section{References}

[1] Y. Kuang, Delay Differential Equations with Applications in Population Dynamics, vol. 191 of Mathematics in Science and Engineering, Academic Press, Boston, Mass, USA, 1993.

[2] F. Bt Ismail and M. B. Suleiman, "The P-stability and Qstability of singly diagonally implicit Runge-Kutta method for delay differential equations," International Journal of Computer Mathematics, vol. 76, no. 2, pp. 267-277, 2000.

[3] O. A. Taiwo and O. S. Odetunde, "On the numerical approximation of delay differential equations by a decomposition method," Asian Journal of Mathematics and Statistics, vol. 3, no. 4, pp. 237243, 2010.

[4] F. Ismail, R. A. Al-Khasawneh, and M. Suleiman, "Numerical treatment of delay differential equations by Runge-Kutta method using Hermite interpolation," Matematika, vol. 18, pp. 79-90, 2002.

[5] Y. S. Hoo, Z. A. Majid, and F. Ismail, "Solving second-order delay differential equations by direct Adams-Moulton method," Mathematical Problems in Engineering, vol. 2013, Article ID 261240, 7 pages, 2013.

[6] H. C. San, Z. A. Majid, and M. Othman, "Solving delay differential equations using coupled block method," in Proceedings of the 4th International Conference on Modeling, Simulation and Applied Optimization (ICMSAO '11), pp. 1-4, IEEE, Kuala Lumpur, Malaysia, April 2011.

[7] H. M. Radzi, Z. A. Majid, F. Ismail, and M. Suleiman, "Two and three point one-step block methods for solving delay differential equations," Journal of Quality Measurement and Analysis, vol. 82, no. 1, pp. 29-41, 1823.

[8] F. Ishak, M. B. Suleiman, and Z. Omar, "Two-point predictorcorrectorblock method for solving delay differential equations," Matematika, vol. 24, no. 2, pp. 131-140, 2008.

[9] S. Z. Ahmad, F. Ismail, N. Senu, and M. Suleiman, "Semi implicit hybrid methods with higher order dispersion for solving oscillatory problems," Abstract and Applied Analysis, vol. 2013, Article ID 136961, 10 pages, 2013.

[10] J. P. Coleman, "Order conditions for class of two-step methods for $y^{\prime \prime}=f(x, y)$," IMA Journal of Numerical Analysis, vol. 23, pp. 197-220, 2003.

[11] Y. D. Jikantoro, F. Ismail, and N. Senu, "Zero-dissipative trigonometrically fitted hybrid method for numerical solution of oscillatory problems," Sains Malaysiana, vol. 44, no. 3, pp. 473482, 2015.

[12] Y. Fang and X. Wu, "A Trigonometrically fitted explicit Numerov-type method for second-order initial value problems with oscillating solutions," Applied Numerical Mathematics, vol. 58, no. 3, pp. 341-351, 2008.

[13] K. Schmitt, "Comparison theorems for second order delay differential equations," Rocky Mountain Journal of Mathematics, vol. 1, no. 3, pp. 459-467, 1971.

[14] G. Ladas and I. P. Stavroulakis, "On delay differential inequalities of first order," Fako de l'Funkcialaj Ekvacioj Japana Matematika Societo. Funkcialaj Ekvaciog. Serio Internacia, vol. 25, no. 1, pp. 105-113, 1982.

[15] B. Singh, "Asymptotic nature on non-oscillatory solutions of $n$th order retarded differential equations," SIAM Journal on Mathematical Analysis, vol. 6, no. 5, pp. 784-795, 1975. 
[16] N. Senu, M. Suleiman, F. Ismail, and M. Othman, "A singly diagonally implicit Runge-Kutta-Nyström method with reduced phase-lag," in Proceedings of the International MultiConference of Engineers and Computer Scientists (IMECS '11), pp. 1489-1494, March 2011.

[17] D. F. Papadopoulos, Z. A. Anastassi, and T. E. Simos, "A modified phase-fitted and amplification-fitted Runge-KuttaNyström method for the numerical solution of the radial Schrödinger equation," Journal of Molecular Modeling, vol. 16, no. 8, pp. 1339-1346, 2010.

[18] J. M. Franco, "A class of explicit two-step hybrid methods for second-order IVPs," Journal of Computational and Applied Mathematics, vol. 187, no. 1, pp. 41-57, 2006.

[19] N. Senu, M. Suleiman, F. Ismail, and M. Othman, "A fourthorder diagonally implicit Runge-Kutta-Nyström method with dispersion of high order," in Proceedings of the 4th International Conference on Applied Mathematics, Simulation, Modeling (ASM '10), pp. 78-82, 2011.

[20] D. F. Papadopoulos, Z. A. Anastassi, and T. E. Simos, "A phase-fitted Runge-Kutta-Nyström method for the numerical solution of initial value problems with oscillating solutions," Computer Physics Communications, vol. 180, no. 10, pp. 18391846, 2009. 


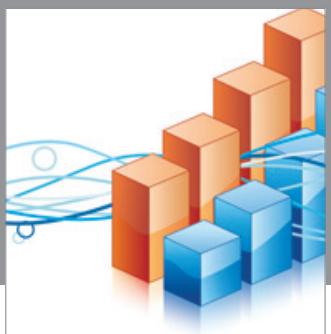

Advances in

Operations Research

vatem alat4

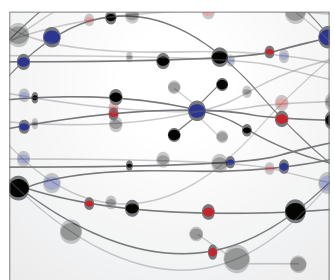

\section{The Scientific} World Journal
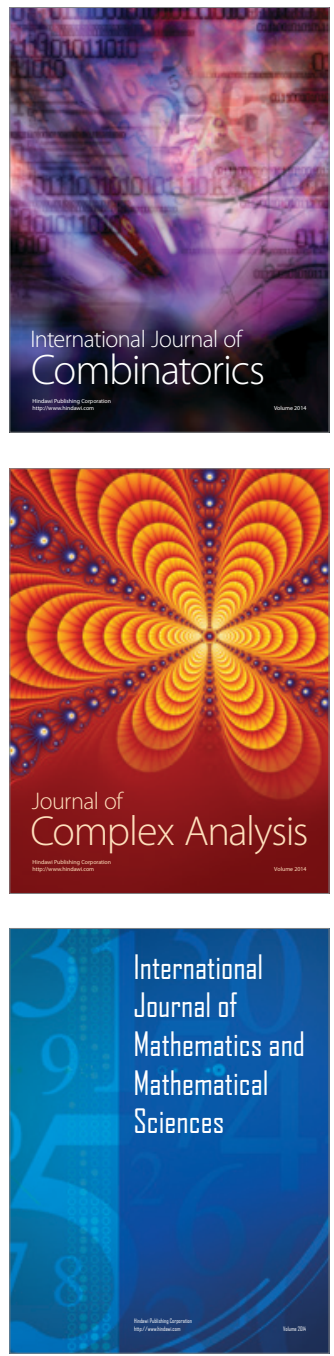
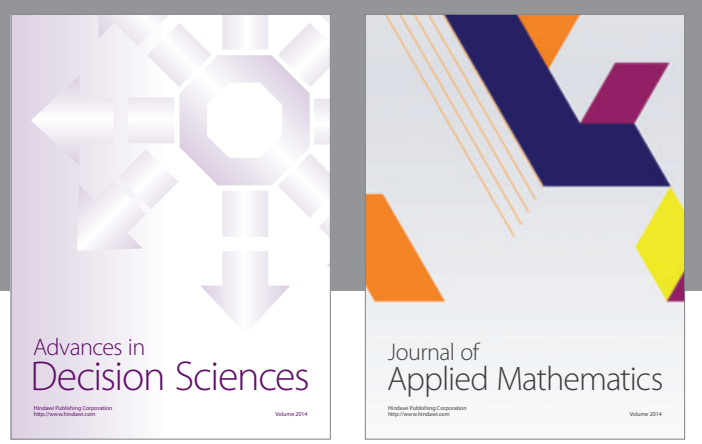

Algebra

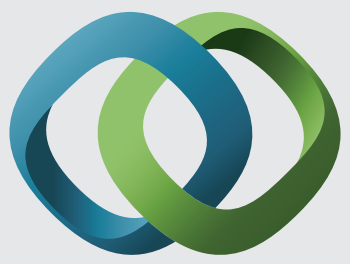

\section{Hindawi}

Submit your manuscripts at

http://www.hindawi.com
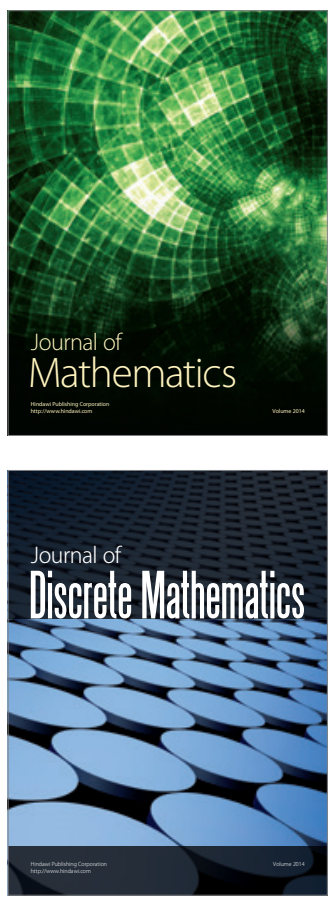

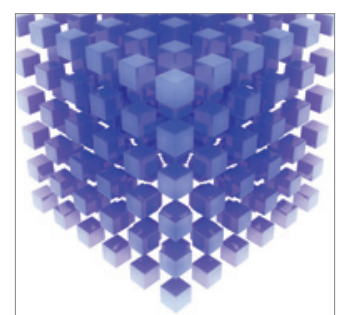

Mathematical Problems in Engineering
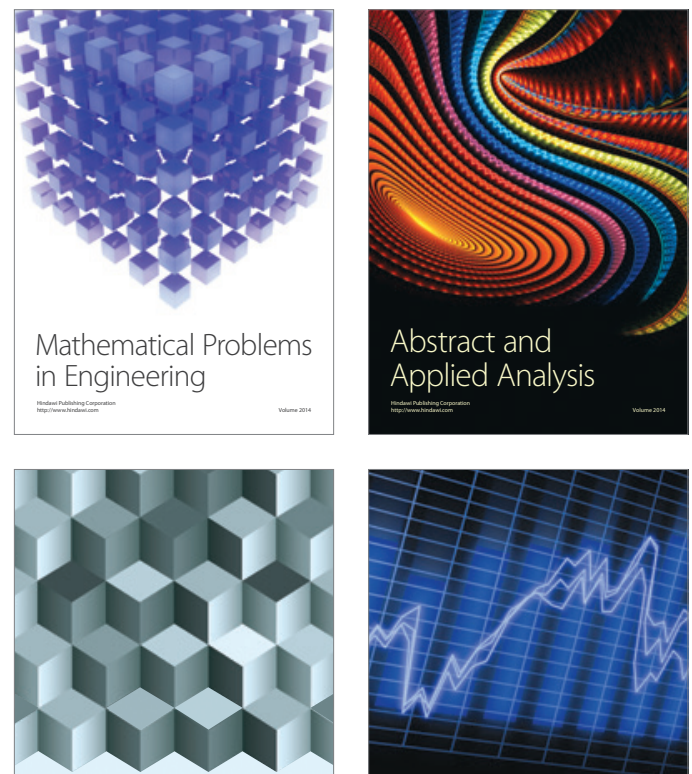

Journal of

Function Spaces

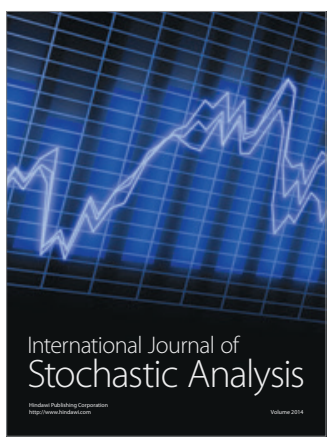

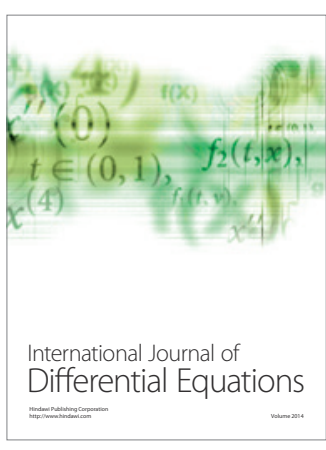
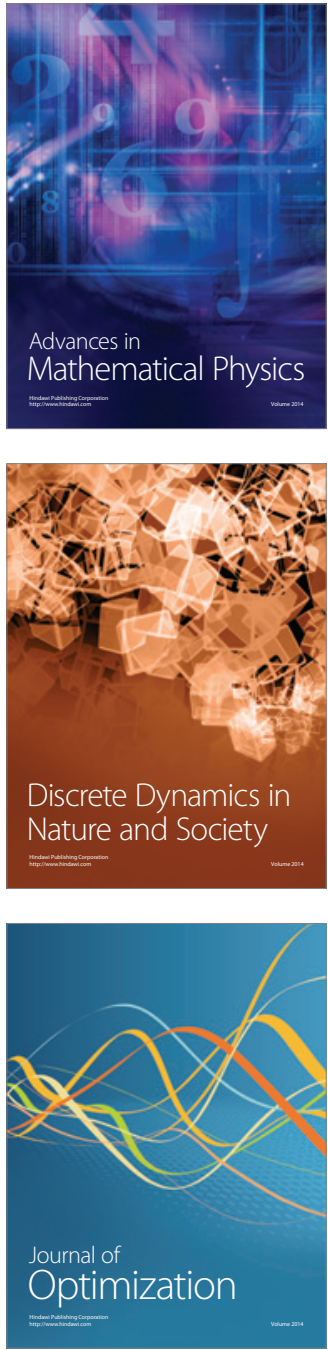